\title{
有线传输技术在通信工程中的应用分析
}

林小冬

黑龙江农是通信有限公司

DOI:10.32629/btr.v2i8.2466

[摘 要] 传输信息通道通常分为有线信道和无限信道形式。一般有线信道传输就是所说的有线传输技术。有线传输技术通 常是通过相应线路的连接开展传输, 线路需要与终端系统和传输系统充分连接,因此为了充分发挥有线传输技术的作用, 本文 阐述了传输技术在通信工程中应用的主要特点就有线传输技术在通信工程中的应用及其发展进行了探讨分析。

[关键词] 传输技术; 特点; 有线传输技术; 通信工程; 应用; 发展方向

目前有线传输主要是通过光缆的形式开展传输, 其具有 信息传输效率高、传输稳定性好等优势, 随着科学技术的不 断发展, 其将得到更加广泛的普及应用, 从而有效提升有线 传输技术水平。

\section{1 传输技术在通信工程中应用的主要特点分析}

1. 1 传输产品体积小。当前我国传输技术产品体积正在 逐年递减, 如网络信号的延伸产品以及光纤收发器等, 虽然 这些产品的外形在缩小, 但是使用起来却越来越灵活。缩小 的传输产品, 能减少空间占用量, 给用户带来诸多便利, 进而 能为生产商和运营商节约诸多成本。运营商在站点延伸或者 扩容期间, 很少增设基础设施, 会将一些产品直接作用在建 筑墙壁上使用, 实现远程控制的同时, 也能缩短建设的实践 周期, 降低投资的成本。传输产品小型化, 能提高产品的性价 比, 也能让支持点的信号更好的传递出去, 实现从E11到 $155 \mathrm{MB}$ 信号的延伸传输, 为让接口交换有着较好的灵活性。小
型化的传输产品在通讯设备使用期间越加广泛, 其中小区的 用户接口、网络接入和集团用户使用等。

1. 2功能多。传输设备小型化的发, 其也开始实现多个独 立设备功能性集成。这样能扩宽传输网络的容量利用效率, 也能缩小光缆纤芯的整体容量占比。为让传输产品的功能更 多, 就要提升产品的全部技术含量, 让传输信号能够更好地 接入到设备内进行传输, 特别是减少分散接入复杂工序, 能 节约现有的使用成本。当前, 受到传输设备整合影响, 在将以 太网信号接入到传输功能后, 具有运营资格的运营商都要通 过互联网实现信号的高效传输, 然后使用互联网信号让传输 接入得以实现。随着我国通信用户的增设, 网络覆盖需求量 正在逐年增大, 所有的相关通信设备也能满足小型号, 多功 能的要求。

\section{2 有线传输技术在通信工程中的应用分析}

2.1 WDM技术的应用。传统通信工程通常运用同步数字
5. 2工程开工前, 组织对桩点、资料进行交接, 严格按照 《城市轨道交通工程测量规范》和《地下铁道工程施工及验 收规范》进行导线点复测和基标测设并按相关检测程序进行 报验, 经检无误后再安排后续测量工作。

5. 3所有中线、水平、高程测量都必须有详细的图表计 算和交底。

5.4 严格执行测量工作双检制。凡接桩复测、施工放线 放样及竣工测量都必须进行双检制, 一个测量队先后用不同 的方法、不同人员测量, 做到复核测量、换手测量, 确保测量 结果准确无误。

5. 5 内业资料必须实行双检制, 做到一人计算、一人复核, 确保内业资料准确无误后签字确认, 方可下发测量资料成果。

5. 6 每次测量作业之前都应按照相关规范的要求对测量 仪器进行检验, 检验无误后方可进行测量工作, 如果仪器检 测不符合相关规范要求, 不得进行测量工作, 以免造成的测 量事故的发生。

\section{6 结语}

随着地铁轨道工程的不断发展, 各类测量技术要求越 来越高, 以及道床类型多样化, 铺轨基标控制测量已不能
满足现有轨道部分类型道床测量施工 (如预制钢弹簧浮置 板) 等。该施工工法使施工工效大幅提升, 促进工期效益的 提高, 经济效果显著。施工工艺相对成熟可靠, 相比传统人 工基桩法具备更快、更经济、对周围环境影响更小的优 势。对施工环境具有更大的适应性, 能实现施工企业降本 增效的同时, 亦满足可持续发展社会节能和地铁降噪减振 的要求。

\section{[参考文献]}

[1]罗小强,金立军,王与娟.地铁轨道工程铺轨基标及CPIII轨道控 制网应用讨论[J].中小企业管理与科技(上旬刊),2014(8):110-112.

[2]敖付勇.CP III 控制网在城市轨道交通建设中的应用探 讨 [J].城市建设理论研究,2013(24):58.

[3]孙柏辉.《铁路轨道工程施工质量验收标准》宣贯要 点[J].铁道标准设计,2004(7):59-64.

[4]卢建康,刘华.高速铁路精密工程测量技术体系的建 立及特点 [J].铁道标准设计,2010(1):70-73.

作业简介：

张䜭航(1994--), 男, 山东滩坊人, 汉族, 本科学历, 从事工 作：轨道工程。 
体系开展信号通信, 但是这种方式不仅局限性高, 传输速度 较慢, 而且成本较高, 无法满足当前通信工程信号通信的具 体需求。因此, 技术人员对通信技术开展了过重改良, 通过有 机的协调同步数字体系和WDM体系来优化资源配置, 同时扩 充了信号通信的容量和整体效果。WDM技术即是利用光纤系 统中的激光传输设备来完成多种波长的激光传输, 它不仅能 够实现信号的多种调节与固定, 还能够实现多种不同波长的 超远距离传输, 而且该技术的传输速度和传输协议相对独立, 不会受限于其他的传输形势。WDM技术应用在通信工程中时, 会利用多种协议来实现信息数据的高速传输, 其信号处理速 度高达 $5 \mathrm{~GB} / \mathrm{S}$, 最低的速度也不会低于 $100 \mathrm{MB} / \mathrm{S}$ 。这种高速的 传输更加适用于超远距离的传输, 而且降低了传统通信工程 中有限传输的整体成本, 使有线传输的信号更具高效性和灵 活性。WDM技术是目前主流的有线传输技术在通信工程中的 应用形式, 它重新定义了同步数字体系信号通信的基本方式, 补充和完善了传统通信体系中存在的不足和缺陷, 有效提升 了数据资料传输的质量和效率, 并降低了数据信息传输的成 本, WDM技术的广泛应用象征着通信工程技术进入了全新的 领域和纪元, 当前通信工程中已经针对WDM技术开展了广泛 的应用和多元化的技术开发, 使其能够为客户提供更便捷的 信息传输服务。

2.2 ASON技术的应用。ASON技术是在传统的WDM网络通 信技术的基础上, 在通信工程上研发的全新技术, 相比于传 统的通信技术而言, 它在业务配置、宽带利用率和保护方式 上更具优势。ASON技术的全称为自动交换光网络技术, 也就 是我们常说的智能光网络。在传输网中引入了信令, 并通过 增加控制平面的方式, 增强了网络连接管理和故障连接的恢 复功能。这种技术支持光学参数的路由计算策略, 能够自动 排除不满足光学参数要求的路径, 并支持重路由和优化时波 长的自动调整, 有效解决了波长冲突的问题, 它有着端到端 的业务自动配置功能, 同时支持拓扑自动发现和Mesh组网保 护, 增强了网络的可生存性, 该技术能够根据客户层信号的 业务等级选取需求的保护等级并支持流量工程控制, 网络可 根据客户层的业务需求开展动态的逻辑设置, 实现了网络资 源的最佳配置。ASON技术拥有先进的业务保护和业务恢复功 能, 只需要选择节点和宿节点, 就能开展多元化的业务参数配 置。其拓扑结构主要是MESH结构, 在传统业务保护的同时, 还 能够实现业务的动态恢复, ASON技术能够根据业务恢复时间 的差异提供多种业务类型, 以此满足客户的不同需求。ASON技 术更加适用于本地网络的通信工程应用, 能够对网络开展系 统的优化和促进作用, 也提升了本地网络维护的便捷性。

3 有线传输技术在通信工程中应用的发展方向分析 3. 1 网络化的发展。随着信息网络技术的快速发展, 数据
传播逐渐从通信向网络化的方向转变, 如QQ、微博、微信、 脸书等。这就表明, 传统的传输方式已经不能满足当代社会 发展的要求, 只能寻求适应当代社会发展的方式才能使有线 传输获得更长久的发展。而网络化发展的理念一经提出, 就 得到了众人的认可, 因为它不仅能在最短的时间内或最小的 空间内为人们传输信息, 而且也保障了信息传输的安全性和 稳定性, 同时, 满足了众人的各种要求, 真正的把有线传输实 现了网络化的发展。

3. 2 多元化发展。有线传输技术已经有越来越多种类的 正在服务人们生产和生活, 不同传输技术具有不同类型的特 点, 并且作用机理也不相同, 这就是不同社会需求下的必然 发展趋势, 因此, 有线传输技术的发展最终将会呈现多元化 形式。例如在实际应用过程当中, 不同波长的信号有时会同 时开展信息传递, 但是单一形式的传输方式并不能达到此种 要求, 只有对原有传输方式开展创新, 才能满足多样需求。传 输技术的多元化发展能够很好地控制信息传递速度, 保证其 传递的一致性, 这样可以防止在传递过程中, 因为信息丢失 而造成不必要的麻烦, 有效保证了信息传递的准确性和可靠 性。同时, 传输技术的多元化发展还有利于控制成本, 减少不 必要的支出。

3. 3 向着商业化的方向发展。商业化发展的有线传输技 术应用过程中, 可以减少不必要的传输环节, 这样就可以进 一步降低传输过程中的成本, 从而提升经济效益。同时, 有线 传输技术的应用, 会让通信工程更具便利性和灵活性, 在技 术拓展过程中, 不仅可以满足大数量的信息传输, 还可以实 现大数量的信息处理, 并且处理效率与质量都十分高, 非常 契合 “信息大爆炸时代” 特点。另一方面, SDH等新型传输技 术的出现, 将会进一步完善光纤传输技术的不足, 让通信工 程涉及范围进一步扩大, 实现大范围的信息传递和处理。

\section{4 结束语}

综上所述, 随着人们生活水平的提升, 人们对通信工程 质量与效率也提出了更多要求。其中有线传输技术作为通信 工程中的重要应用技术受到越来越多人的广泛关注, 因此对 有线传输技术在通信工程中的应用进行分析具有重要意义。

\section{[参考文献]}

[1]纪长亮.传输技术在通信工程中的应用 [J].科学导 报,2019,(02):163.

[2]周东来.论传输技术在通信工程中的应用及发展方向 [J].电脑迷,2017,(12):72.

[3]柴青辰.传输技术在通信工程中的应用及发展分析 [J].通讯世界,2017,(12):72.

[4]华月铭.试论传输技术在通信工程中的应用及发展方 向[J].科技经济导刊,2017(3):25. 